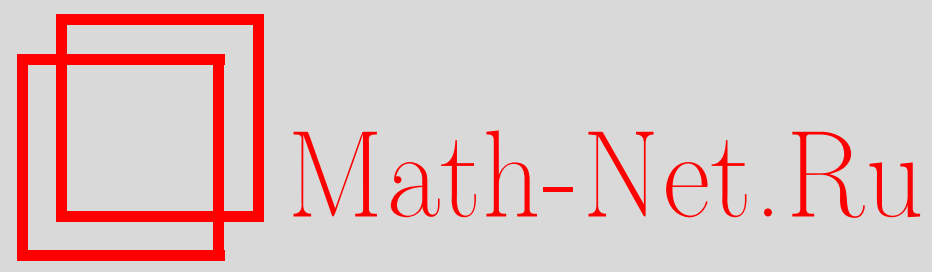

В. Б. Алексеев, Р. Р. Омаров, О приближении максимальнонелинейных булевых функций почти линейными функциями, Дискрет. матем., 2012, том 24, выпуск 3, 73-81

DOI: https://doi.org/10.4213/dm1199

Использование Общероссийского математического портала Math-Net.Ru подразумевает, что вы прочитали и согласны с пользовательским соглашением http://www . mathnet.ru/rus/agreement

Параметры загрузки:

IP: 54.157 .27 .8

26 апреля 2023 г., $16: 23: 02$ 


\title{
О приближении максимально-нелинейных булевых функций почти линейными функциями
}

\author{
() 2012 г. В. Б. Алексеев, Р. Р. Омаров
}

\begin{abstract}
Известно, что максимальное расстояние между булевой функций от $2 n$ переменных и классом аффинных булевых функций от $2 n$ переменных равно $2^{2 n-1}-2^{n-1}$. В работе исследуется расстояние между классом максимально-нелинейных булевых функций от $2 n$ переменных и классом булевых функций от $2 n$ переменных, у которых в полиноме Жегалкина присутствует не более $k$ нелинейных слагаемых. Показано, что при любом фиксированном $k$ и $n \rightarrow \infty$ это расстояние равно $2^{2 n-1}-3^{k} \cdot 2^{n-1}+o\left(2^{n-1}\right)$.

Работа поддержана Российским фондом фундаментальных исследований, проект 10-01-00475-a.
\end{abstract}

В качестве одного из криптографических параметров булевых функций часто рассматривается нелинейность, характеризующая удаленность данной булевой функции от аффинных (иногда их называют линейными) функций. Это связано с тем, что аффинность булевой функции, используемой в криптографической системе, позволяет производить успешную атаку на такую систему и даже ее близость к аффинным функциям облегчает поиск слабостей в таких системах.

Обзор имеющихся результатов о нелинейности булевых функций можно найти в книге [1]. В частности, там описаны некоторые классы булевых функций с максимальным значением нелинейности. Качественно это означает, что такие функции плохо приближаются аффинными функциями. Однако в принципе может оказаться, что данную функцию с максимальным значением нелинейности удастся хорошо приблизить почти аффинной булевой функцией, то есть булевой функцией с небольшим числом нелинейных слагаемых в ее полиноме Жегалкина. Вопрос о возможностях такой ситуации и исследуется в данной работе.

Приведем необходимые определения.

Пусть $n$ - произвольное натуральное число. Через $V_{n}$ будем обозначать векторное пространство наборов длины $n$ с компонентами из $\{0,1\}$ с операцией $\oplus$ покоординатного сложения векторов по модулю 2.

Определение 1. Пусть $f-$ булева функция от $n$ переменных, то есть $f: V_{n} \rightarrow\{0,1\}$. Ее весом $w t(f)$ будем называть количество наборов, на которых функция равна 1. Так как у функции могут быть фиктивные переменные, то в некоторых случаях для определенности мы будем писать $w t_{n}(f)$, чтобы явно указать, на каком множестве переменных рассматривается данная функция. 
Определение 2. Пусть $f, g$ - булевы функции от $n$ переменных. Расстоянием от булевой функции $f$ до булевой функции $g$ называется величина

$$
\operatorname{dist}(f, g)=w t(f \oplus g),
$$

то есть количество наборов, на которых значения $f$ и $g$ различаются. Расстоянием от $f$ до множества $M$ булевых функций от $n$ переменных назовем величину

$$
\operatorname{dist}(f, M)=\min _{g \in M} \operatorname{dist}(f, g) .
$$

Под расстоянием между двумя множествами булевых функций $M$ и $N$ будем понимать

$$
\operatorname{dist}(M, N)=\min _{\substack{g \in M \\ h \in N}} \operatorname{dist}(g, h) .
$$

Определение 3. Пусть $x \in V_{n}, y \in V_{n}$. Через $\langle x, y\rangle$ будем обозначать скалярное произведение $x$ и $y$ :

$$
\langle x, y\rangle=x_{1} y_{1} \oplus \ldots \oplus x_{n} y_{n}
$$

где $\oplus-$ сложение по модулю 2.

Определение 4. Булева функция $f$ от $n$ переменных называется аффинной, если существуют $a=\left(a_{1}, \ldots, a_{n}\right) \in V_{n}$ и $c \in\{0,1\}$ такие, что

$$
f(x)=\langle a, x\rangle \oplus c=a_{1} x_{1} \oplus \ldots \oplus a_{n} x_{n} \oplus c .
$$

Множество всех аффинных булевых функций от $n$ переменных будем обозначать $A_{n}$.

Отметим, что для любой аффинной булевой функции $f(x)$ от $n$ переменных, отличной от константы, справедливо равенство

$$
w t(f(x))=2^{n-1} .
$$

Определение 5. Расстояние $\operatorname{dist}\left(f, A_{n}\right)$ от булевой функции $f(x)$ от $n$ переменных до множества $A_{n}$ аффинных булевых функций называется нелинейностью функции $f(x)$ и обозначается через $N_{f}$.

Лемма 1 ([1, с.234]). Для любой булевой функции $f(x)$ от $n$ переменных справедливо неравенство

$$
N_{f} \leqslant 2^{n-1}-2^{n / 2-1}
$$

Для четных $n$ эта оценка достижима.

Определение 6. Булевы функции $f(x)$, для которых $N_{f}$ равно максимально возможному значению среди всех функций от $n$ переменных, называют максимально-нелинейными функциями (при четном $n$ этот класс также называют классом бент-функций). Множество всех максимально-нелинейных булевых функций от $2 n$ переменных будем обозначать $B_{2 n}$.

Определение 7. Через $\mathrm{AF}_{n}^{k}$ будем обозначать класс всех булевых функций от $n$ переменных вида $X_{1} \oplus \ldots \oplus X_{k} \oplus l(x)$, где каждое $X_{j}$ - конъюнкция некоторых переменных или 0 , а $l(x) \in A_{n}$. При $k=0$ полагаем $\mathbb{A E}_{n}^{0}=A_{n}$. 
Введем функцию

$$
\rho_{k}(U)=\operatorname{dist}\left(U, \mathbb{A E}_{2 n}^{k}\right),
$$

где $U-$ некоторое множество функций от $2 n$ переменных. В данной статье мы исследуем поведение функции $\rho_{k}\left(B_{2 n}\right)$ при произвольном фиксированном $k$.

Одним из широко известных классов максимально-нелинейных булевых функций является класс Мэйорана-МакФарланда.

Определение 8. Пусть $x=\left(x_{1}, \ldots, x_{n}\right)$ и $y=\left(y_{1}, \ldots, y_{n}\right)$. Определим класс МэйоранаМакФарланда определяется как класс всех булевых функций $f(x, y)$ от $2 n$ переменных вида

$$
f(x, y)=\langle\pi(y), x\rangle \oplus \Phi(y),
$$

где $\pi-$ произвольная подстановка на множестве $V_{n}$, а $\Phi(y)-$ произвольная булева функция от $n$ переменных. Будем обозначать его через $M_{2 n}$.

Известно (см., например, [1]), что все функции из класса Мэйорана-МакФарланда являются максимально-нелинейными. Ранее авторами $[2,3]$ было показано, что

$$
\rho_{k}\left(M_{2 n}\right)>2^{2 n-1}-3^{k} \cdot 2^{n-1}, \quad k=1,2 .
$$

Оказывается, что эта оценка справедлива для всех $k$ и даже для всего класса $B_{2 n}$.

Теорема 1. Пусть $B_{2 n}-$ множество всех максимально-нелинейных булевых функций от $2 n$ переменных, тогда для любого натурального $k$

$$
\rho_{k}\left(B_{2 n}\right) \geqslant 2^{2 n-1}-3^{k} \cdot 2^{n-1} .
$$

Доказательство. Пусть $f(x)$ - произвольная максимально-нелинейная булева функция от $2 n$ переменных, а

$$
g(x)=X_{1} \oplus \ldots \oplus X_{k} \oplus\langle a, x\rangle \oplus c
$$

- произвольная функция из класса $\mathrm{AF}_{2 n}^{k}$, где каждое $X_{i}$ - конъюнкция некоторых переменных или 0. Требуется доказать, что

$$
\operatorname{dist}(f(x), g(x)) \geqslant 2^{2 n-1}-3^{k} \cdot 2^{n-1} .
$$

Обозначим

$$
\begin{aligned}
f^{\prime}(x) & =f(x) \oplus\langle a, x\rangle \oplus c, \\
g^{\prime}(x) & =X_{I_{1}} \oplus \ldots \oplus X_{I_{k}} .
\end{aligned}
$$

Заметим, что вес суммы двух функций $h_{1}(x) \oplus h_{2}(x)$ можно вычислить, используя следующую процедуру. Сначала мы рассматриваем $h_{1}(x)$ и ее вес и замечаем, что при переходе от $h_{1}(x)$ к $h_{1}(x) \oplus h_{2}(x)$ в тех точках, где $h_{2}(x)=0$, ничего не меняется, а в тех точках, где $h_{2}(x)=1$, в вес добавляется 1 , если $h_{1}(x)=0$, и вычитается 1 , если $h_{1}(x)=1$. Поэтому

$$
\begin{aligned}
\operatorname{dist}(f(x), g(x)) & =w t(f(x) \oplus g(x))=w t\left(f^{\prime}(x) \oplus g^{\prime}(x)\right) \\
& =w t\left(f^{\prime}(x)\right)+\sum_{\substack{x \in V_{2 n} \\
g^{\prime}(x)=1}}(-1)^{f^{\prime}(x)} .
\end{aligned}
$$


Условие $g^{\prime}(x)=1$ означает, что нечетное число слагаемых $X_{l}$ обращается в 1 на наборе $x$. Докажем следующую лемму, которая является вариантом известного принципа включения-исключения.

Лемма 2. Пусть задано семейство подмножеств $A_{1}, A_{2}, \ldots, A_{k}$ некоторого множества, и пусть $D$ - множество всех элементов, содержащихся в нечетном количестве подмножеств этого семейства. Пусть на множестве элементов из $A_{1} \cup A_{2} \cup \ldots \cup A_{k}$ задана весовая функиия $w(x)$. Тогда

$$
\sum_{x \in D} w(x)=\sum_{t=1}^{k}(-2)^{t-1} \sum_{\left\{i_{1}, i_{2}, \ldots, i_{t}\right\} \subseteq\{1,2, \ldots, k\}} \sum_{x \in A_{i_{1}} \cap \ldots \cap A_{i_{t}}} w(x) .
$$

Доказательство. Пусть элемент $x$ содержится ровно в $p$ подмножествах из $A_{1}, \ldots, A_{k}$. Тогда его вес $w(x)$ будет присутствовать в правой части с коэффициентом

$$
\begin{aligned}
\sum_{t=1}^{k}(-2)^{t-1}\left(\begin{array}{l}
p \\
t
\end{array}\right) & =\frac{1}{2}\left[1-\sum_{t=0}^{k}\left(\begin{array}{l}
p \\
t
\end{array}\right)(-2)^{t}\right]=\frac{1-(1-2)^{p}}{2} \\
& =\frac{1-(-1)^{p}}{2}= \begin{cases}0 & \text { если } p \text { четно, } \\
1 & \text { если } p \text { нечетно. }\end{cases}
\end{aligned}
$$

Таким образом, в правой части каждый вес $w(x)$ будет присутствовать с тем же коэффициентом, что и в левой части. Лемма доказана.

Учитывая лемму 2 и соотношение, полученное перед ней, получаем, что

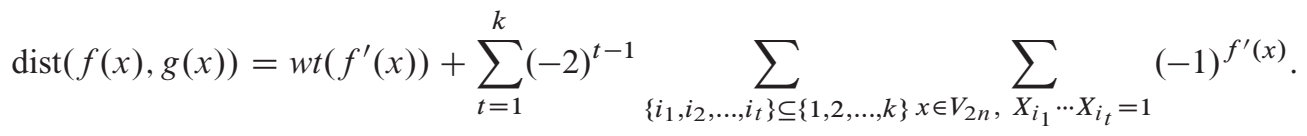

Лемма 3. Пусть $A \subseteq\{1,2, \ldots, 2 n\}$, и пусть $X_{A}$ - конъюнкция всех переменньх $x_{i}$ таких, что $i \in A$. Пусть $h(x)$ - максимально-нелинейная функция от $2 n$ переменных. Тогда

$$
\left|\sum_{x \in V_{2 n}, X_{A}=1}(-1)^{h(x)}\right| \leqslant 2^{n}
$$

Доказательство. Пусть $\xi^{A} \in V_{2 n}$ - такой вектор, что $\xi_{i}^{A}=1$, если $i \in A$, и $\xi_{i}^{A}=0$, если $i \notin A$. Пусть

$$
L_{A}=\left\{x \in V_{2 n}: x_{i}=0 \text { для всех } i \in A\right\} .
$$

Заметим, что $L_{A}$ есть линейное подпространство в $V_{2 n}$ размерности $2 n-|A|$ и

$$
\left\{x: x \in V_{2 n}, X_{A}=1\right\}=\left\{\xi^{A} \oplus x: x \in L_{A}\right\}=\xi^{A}+L_{A} .
$$

Пусть $L_{A}^{\perp}-$ ортогональное дополнение к подпространству $L_{A}$ в $V_{2 n}$.

Так как по условию $h(x)$ - максимально-нелинейная функция от $2 n$ переменных, то для нее существует такая функция $\tilde{h}(x)$ также от $2 n$ переменных (дуальная функция к 
$h(x))$, что для $h(x)$ и $\tilde{h}(x)$ выполняется равенство (лемма 6.22 при $b=0$ в [1, с. 240]):

$$
\begin{aligned}
\sum_{x \in V_{2 n}, X_{A}=1}(-1)^{h(x)} & =\sum_{x \in \xi^{A}+L_{A}}(-1)^{h(x)}=2^{\operatorname{dim} L_{A}-n} \sum_{x \in L_{A}^{\perp}}(-1)^{\tilde{h}(x) \oplus\left\langle\xi^{A}, x\right\rangle} \\
& =2^{n-|A|} \sum_{x \in L_{A}^{\perp}}(-1)^{\tilde{h}(x) \oplus\left\langle\xi^{A}, x\right\rangle} .
\end{aligned}
$$

Так как размерность $L_{A}^{\perp}$ равна $|A|$, то мощность $L_{A}^{\perp}$ равна $2^{|A|}$ и

$$
\left|\sum_{x \in L_{A}^{\perp}}(-1)^{\tilde{h}(x) \oplus\left\langle\xi^{A}, x\right\rangle}\right| \leqslant 2^{|A|} .
$$

Поэтому

$$
\left|\sum_{x \in V_{2 n}, X_{A}=1}(-1)^{h(x)}\right| \leqslant 2^{n-|A|} \cdot 2^{|A|}=2^{n} .
$$

Лемма доказана.

Так как

$$
f^{\prime}(x)=f(x) \oplus\langle a, x\rangle \oplus c,
$$

то для любой функции $l(x)$ получаем, что

$$
\operatorname{dist}(f(x), l(x))=\operatorname{dist}\left(f^{\prime}(x), l(x) \oplus\langle a, x\rangle \oplus c\right) .
$$

Поэтому

$$
\operatorname{dist}\left(f^{\prime}(x), A_{2 n}\right)=\operatorname{dist}\left(f(x), A_{2 n}\right) .
$$

Поскольку $f(x)$ - максимально-нелинейная функция по условию, то и $f^{\prime}(x)$ - максимально-нелинейная функция. Тогда по лемме 3 получаем, что

$$
\begin{aligned}
& \mid \sum_{t=1}^{k}(-2)^{t-1} \sum_{\left\{i_{1}, i_{2}, \ldots, i_{t}\right\} \subseteq\{1,2, \ldots, k\}} \sum_{x \in V_{2 n}, X_{i_{1}} \cdots X_{i_{t}}=1}(-1)^{f^{\prime}(x)} \mid \\
& \leqslant 2^{n} \sum_{t=1}^{k} 2^{t-1}\left(\begin{array}{l}
k \\
t
\end{array}\right)=2^{n-1}\left[(1+2)^{k}-1\right]=\left(3^{k}-1\right) 2^{n-1} .
\end{aligned}
$$

Так как $f^{\prime}(x)$ - максимально-нелинейная функция от $2 n$ переменных, то

$$
w t\left(f^{\prime}(x)\right)=\operatorname{dist}\left(f^{\prime}(x), 0\right) \geqslant 2^{2 n-1}-2^{n-1} .
$$

С использованием соотношения, полученного перед леммой 3 , находим, что

$$
\operatorname{dist}(f(x), g(x)) \geqslant 2^{2 n-1}-2^{n-1}-\left(3^{k}-1\right) 2^{n-1}=2^{2 n-1}-3^{k} 2^{n-1} .
$$

В силу произвольности выбора функций $f(x)$ и $g(x)$, заключаем, что

$$
\rho_{k}\left(B_{2 n}\right) \geqslant 2^{2 n-1}-3^{k} \cdot 2^{n-1} \text {. }
$$

Теорема 1 доказана. 
Так как $M_{2 n} \subseteq B_{2 n}$, то

$$
\rho_{k}\left(M_{2 n}\right) \geqslant \rho_{k}\left(B_{2 n}\right) \geqslant 2^{2 n-1}-3^{k} \cdot 2^{n-1} .
$$

Возникает вопрос, насколько эти оценки точны. Ответ на него дает следующая теорема.

Теорема 2. При любом фиксированном $k$ и $n \rightarrow \infty$ верна оценка

$$
\rho_{k}\left(M_{2 n}\right)=2^{2 n-1}-3^{k} \cdot 2^{n-1}+o\left(2^{n-1}\right) .
$$

Доказательство. Для доказательства построим пример функций $f(x, y) \in M_{2 n}$ и $g(x, y) \in \mathbb{A}_{2 n}^{k}, x, y \in V_{n}$, для которых

$$
\operatorname{dist}(f, g)=2^{2 n-1}-3^{k} \cdot 2^{n-1}+o\left(2^{n-1}\right) .
$$

Пусть $n=p k+t, 0 \leqslant t<k$. Рассмотрим функцию

$$
v\left(x_{1}, \ldots, x_{p}, y_{1}, \ldots, y_{p}\right)=\bigoplus_{i=1}^{p} x_{i} y_{i} \oplus y_{1} \oplus \ldots \oplus y_{p} \oplus \operatorname{sg}\left(y_{1}, \ldots, y_{p}\right),
$$

где $\operatorname{sg}(0, \ldots, 0)=0$ и $\operatorname{sg}\left(y_{1}, \ldots, y_{p}\right)=1$, если $\left(y_{1}, \ldots, y_{p}\right) \neq(0, \ldots, 0)$.

Лемма 4. Для веса любой функциии вида

$$
w\left(x_{1}, \ldots, x_{m}, y_{1}, \ldots, y_{m}\right)=\langle x, y\rangle \oplus \Phi(y),
$$

где $Ф(y)$ - произвольная функция, справедливо равенство

$$
w t_{2 m}(w(x, y))=2^{2 m-1}-(-1)^{\Phi(\tilde{0})} 2^{m-1} .
$$

Доказательство. При любой фиксации всех переменных $y$ в функции $w$, кроме $y=(0, \ldots, 0)$, получаем аффинную функцию от $n$ переменных, отличную от константы, которая равна 1 на половине своих наборов. При $y=(0, \ldots, 0)$ получаем константу $\Phi(\tilde{0})$. Поэтому вес $w t_{2 m}(w(x, y))$ отличается от половины всех наборов на $(1 / 2) 2^{m}$ вверх или вниз в зависимости от значения $\Phi(\tilde{0})$ и равен $2^{2 m-1}-(-1)^{\Phi(\tilde{0})} \cdot 2^{m-1}$. Лемма доказана.

Лемма 5. Справедливо равенство

$$
w t_{2 p}\left(v\left(x_{1}, \ldots, x_{p}, y_{1}, \ldots, y_{p}\right) \oplus x_{1} \cdot x_{2} \cdots x_{p}\right)=2^{2 p-1}-\left(3 \cdot 2^{p-1}-2\right) .
$$

Доказательство. С использованием леммы 4 нетрудно получить,что

$$
\begin{aligned}
w t_{2 p}( & \left.v\left(x_{1}, \ldots, x_{p}, y_{1}, \ldots, y_{p}\right) \oplus x_{1} \cdot x_{2} \cdots x_{p}\right) \\
= & w t_{2 p}\left(v\left(x_{1}, \ldots, x_{p}, y_{1}, \ldots, y_{p}\right)\right)+w t_{2 p}\left(x_{1} \cdots x_{p}\right) \\
& \quad-2 w t_{2 p}\left(x_{1} \cdot x_{2} \cdots x_{p} \cdot v\left(x_{1}, \ldots, x_{p}, y_{1}, \ldots, y_{p}\right)\right) \\
= & 2^{2 p-1}-2^{p-1}+2^{p}-2 w t_{p}\left(\operatorname{sg}\left(y_{1}, \ldots, y_{p}\right)\right) \\
= & 2^{2 p-1}+2^{p-1}-2\left(2^{p}-1\right) \\
= & 2^{2 p-1}-\left(3 \cdot 2^{p-1}-2\right) .
\end{aligned}
$$

Лемма доказана. 
Пусть переменные $x=\left(x_{1}, \ldots, x_{n}\right), y=\left(y_{1}, \ldots, y_{n}\right)$ определены на $V_{n}$, и пусть

$$
\begin{aligned}
\tilde{X}_{j} & =\left(x_{p(j-1)+1}, x_{p(j-1)+2}, \ldots, x_{p j}\right), \\
\tilde{Y}_{j} & =\left(y_{p(j-1)+1}, y_{p(j-1)+2}, \ldots, y_{p j}\right), \quad j=1,2, \ldots, k .
\end{aligned}
$$

Определим

$$
f(x, y)=\bigoplus_{j=1}^{k} v\left(\tilde{X}_{j}, \tilde{Y}_{j}\right) \oplus \bigoplus_{i=p k+1}^{n} x_{i} y_{i}
$$

Тогда

$$
f(x, y)=\langle x, y\rangle \oplus \Phi(y)
$$

для некоторой функции $\Phi(y)$ и, следовательно, $f(x, y) \in M_{2 n}$. Пусть $K_{j}-$ конъюнкция всех переменных из $\tilde{X}_{j}$. Положим

$$
g(x, y)=K_{1} \oplus K_{2} \oplus \ldots \oplus K_{k} .
$$

Тогда $g(x, y) \in \mathbb{A E}_{2 n}^{k}$. Рассмотрим $\operatorname{dist}(f(x, y), g(x, y))$. Мы видим, что

$$
\begin{aligned}
\operatorname{dist}(f(x, y), g(x, y)) & =w t_{2 n}(f(x, y) \oplus g(x, y)) \\
& =w t_{2 n}\left(\bigoplus_{j=1}^{k}\left(v\left(\tilde{X}_{j}, \tilde{Y}_{j}\right) \oplus K_{j}\right) \oplus \bigoplus_{i=p k+1}^{n} x_{i} y_{i}\right) .
\end{aligned}
$$

В силу леммы 5,

$$
w t_{2 p}\left(v\left(\tilde{X}_{j}, \tilde{Y}_{j}\right) \oplus K_{j}\right)=2^{2 p-1}-\left(3 \cdot 2^{p-1}-2\right) .
$$

Положим

$$
\begin{aligned}
& D=2^{2 p-1}-\left(3 \cdot 2^{p-1}-2\right), \\
& E=2^{2 p}-D=2^{2 p-1}+\left(3 \cdot 2^{p-1}-2\right) .
\end{aligned}
$$

Лемма 6. Справедливо равенство

$$
w t_{2 p k}\left(\bigoplus_{j=1}^{k}\left(v\left(\tilde{X}_{j}, \tilde{Y}_{j}\right) \oplus K_{j}\right)\right)=2^{2 p k-1}-\frac{1}{2}\left(3 \cdot 2^{p}-4\right)^{k}
$$

Доказательство. Функция $\bigoplus_{j=1}^{k}\left(v\left(\tilde{X}_{j}, \tilde{Y}_{j}\right) \oplus K_{j}\right)$ равна 1 , если нечетное число слагаемых обращается в 1. Заметим, что все слагаемые зависят от разных переменных. Поэтому

$$
\begin{aligned}
w t_{2 p k}\left(\bigoplus_{j=1}^{k}\left(v\left(\tilde{X}_{j}, \tilde{Y}_{j}\right) \oplus K_{j}\right)\right) & =\sum_{i \text { is odd }}\left(\begin{array}{c}
k \\
i
\end{array}\right) D^{i} E^{k-i} \\
& =\frac{1}{2}\left((D+E)^{k}-(E-D)^{k}\right) \\
& =\frac{1}{2}\left(2^{2 p k}-\left(3 \cdot 2^{p}-4\right)^{k}\right) \\
& =2^{2 p k-1}-\frac{1}{2}\left(3 \cdot 2^{p}-4\right)^{k} .
\end{aligned}
$$

Лемма доказана. 
Обозначим

$$
\begin{aligned}
\bigoplus_{j=1}^{k}\left(v\left(\tilde{X}_{j}, \tilde{Y}_{j}\right) \oplus K_{j}\right) & =\varphi_{1}, \\
\bigoplus_{i=p k+1}^{n} x_{i} y_{i} & =\varphi_{2} .
\end{aligned}
$$

Заметим, что $\varphi_{1}$ и $\varphi_{2}$ зависят от разных переменных, и по леммам 6 и 4

$$
\begin{aligned}
w t_{2 p k}\left(\varphi_{1}\right) & =2^{2 p k-1}-\frac{1}{2}\left(3 \cdot 2^{p}-4\right)^{k}, \\
w t_{2 n-2 p k}\left(\varphi_{2}\right) & =2^{2 n-2 p k-1}-2^{n-p k-1} .
\end{aligned}
$$

Тогда функция $\varphi_{1}$ как функция от $2 p k$ переменных равна 0 на $2^{2 p k-1}+\frac{1}{2}\left(3 \cdot 2^{p}-4\right)^{k}$ наборах, а функция $\varphi_{2}$ как функция от $2 n-2 p k$ переменных равна 0 на $2^{2 n-2 p k-1}+$ $2^{n-p k-1}$ наборах. Отсюда получаем, что

$$
\begin{aligned}
\operatorname{dist}(f(x, y), g(x, y))= & w t_{2 n}\left(\varphi_{1} \oplus \varphi_{2}\right) \\
= & \left(2^{2 p k-1}-\frac{1}{2}\left(3 \cdot 2^{p}-4\right)^{k}\right)\left(2^{2 n-2 p k-1}+2^{n-p k-1}\right) \\
& \quad+\left(2^{2 p k-1}+\frac{1}{2}\left(3 \cdot 2^{p}-4\right)^{k}\right)\left(2^{2 n-2 p k-1}-2^{n-p k-1}\right) \\
= & 2^{2 n-1}-\left(3 \cdot 2^{p}-4\right)^{k} 2^{n-p k-1} \\
= & 2^{2 n-1}-2^{n-p k-1} 2^{p k} 3^{k}\left(1-\frac{4}{3 \cdot 2^{p}}\right)^{k} \\
= & 2^{2 n-1}-2^{n-1} 3^{k}\left(1-\frac{4}{3 \cdot 2^{p}}\right)^{k} \\
= & 2^{2 n-1}-3^{k} 2^{n-1}+o\left(2^{n-1}\right),
\end{aligned}
$$

так как $k$ есть константа и $p \rightarrow \infty$. Теорема 2 доказана.

С учетом того, что

$$
\rho_{k}\left(B_{2 n}\right) \leqslant \rho_{k}\left(M_{2 n}\right),
$$

из теорем 1 и 2 получаем следующие утверждения.

Теорема 3. При любом фиксированном $k$ u $n \rightarrow \infty$ справедлива оцеенка

$$
\rho_{k}\left(B_{2 n}\right)=2^{2 n-1}-3^{k} \cdot 2^{n-1}+o\left(2^{n-1}\right) .
$$

Теорема 4. При любом фиксированном $k$ u $n \rightarrow \infty$ справедлива оценка

$$
\rho_{k}\left(M_{2 n}\right)=2^{2 n-1}-3^{k} \cdot 2^{n-1}+o\left(2^{n-1}\right) .
$$




\section{Список литературы}

1. Логачев О. А., Сальников А. А., Ященко В. В., Булевы функциии в теории кодирования и криптологии. МЦНМО, Москва, 2004.

2. Алексеев В. Б., Омаров Р. Р., Исследование одного параметра булевых функций, близкого к нелинейности. Научные ведомости Белгородского государственного университета (2009) 15 (70), №12/1, 81-87.

3. Алексеев В. Б., Омаров Р. Р., О расстояниях от максимально-нелинейных булевых функций до почти аффинных функций. В сб.: Материаль XVI Международной конференции «Проблемь теоретической кибернетики». Нижегородский университет, Нижний Новгород, 2011, с. 24-28.

Статья поступила 8.06.2012. 